Journal of Agricultural Sciences
(Tarim Bilimleri Dergisi)

\title{
Catching Performance and Catching Efficiency of Siliconized Baits in Handline Fishery
}

\author{
Celalettin AYDIN ${ }^{a^{*}} \mathbb{D}$, Akın Türker İLKYAZ ${ }^{a}$ (D)

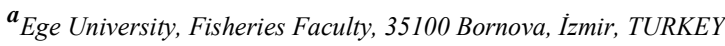 \\ ARTICLE INFO \\ Research Article \\ Corresponding Author: Celalettin AYDIN, E-mail: caydina@gmail.com \\ Received: 19 August 2019 / Revised: 05 February 2020 / Accepted: 03 March 2020 / Online: 31 May 2021
}

\section{ABSTRACT}

The present study investigated the catching performance and catching efficiency of natural mud shrimp (alive) (Upogebia pusilla), siliconized mud shrimp and siliconized pellet in handline fishing. The trials were performed in Kıyıkışlacık Village of Muğla province. The main body of the handline is $\varnothing 0.50 \mathrm{~mm}$, leader and snood are $\varnothing 0.27-0.30 \mathrm{~mm}$, the snood length and distance are $10 \mathrm{~cm}$ and $20 \mathrm{~cm}$, respectively. Each handline has 3 hooks and the hook type is 4 no straight. Catches from natural mud shrimp, siliconized shrimp and siliconized pellet bait retained separately, sorted by species and weighed as. $0.01 \mathrm{~g}$ sensitivity and measured as the total length. Totally 590 individuals from 30 different species in total were caught including; 28 bony fish $(93.33 \%)$, and 2 cephalopods $(0.66 \%)$. While natural mud shrimp captured the $50.7 \%$ of fish, $44.7 \%$ and $4.6 \%$ were caught with siliconized mud shrimp and siliconized pellet, respectively. CPUE values of natural mud shrimp, siliconized shrimp and silicon pellet were calculated as $1.57 \mathrm{n} / \mathrm{h}, 1.38 \mathrm{n} / \mathrm{h}$ and $0.14 n / h$, respectively. In addition, YPUE values were determined as $121.84 \mathrm{~g} / \mathrm{h}, 137.73 \mathrm{~g} / \mathrm{h}$ and $7.62 \mathrm{~g} / \mathrm{h}$ for natural mud shrimp, siliconized shrimp and silicon pellet, respectively. Despite the fact that the number of individuals that a live mud shrimp catch in a unit of time is high, it was found out that the weight is more in a unit of time when silicone mud shrimp is used.

It was concluded that using of siliconized mud shrimp has a high capacity of catching performance and catching efficiency and can be used as bait when natural mud shrimp cannot be utilized as alive or unable to be supplied, due to the weather conditions and time restrictions.

Keywords: Handline fishing, Mud shrimp, Upogebia pusilla, Siliconized bait, Pellet feed, Aegean sea

(C) Ankara University, Faculty of Agriculture

\section{Introduction}

The history of handline fishing dates back to old times (Huse \& Fernö 1990). Today, it is commonly used around the world both for commercial and amateur purposes. It has a low cost for high catching efficiency. Due to both easy make and uses it can be used from small lakes to the oceans.

The most important factors that affect on catching performance in handline fishery are hooks and baits. While the size and shape of the hook have distinct characteristics for target species, the efficiency of the bait may change according to the catching area, season and target species etc. Fishes tend to prefer baits that are existed in their habitat which are common for them. Therefore, fishers use these baits as much as possible. However, reasons such as the incapability to provide an ideal bait and the high-cost lead fishers to come up with alternative solutions.

There are some studies conducted on handline fishing in Turkey. Kaykaç et al. (2003) presented catching efficiency of the cross and straight hooks. Akamca \& Kiyaga (2009) investigated the prey-predator relationship of sea bass (Dicentrarchus labrax) line fishing in İskenderun Bay, Yumurtalı Cove. Aydin (2011) compared the impacts of razor clam (Solen vagina) and sardine (Sardina pilchardus) on annular seabream (Diplodus annularis), picarel (Spicara flexuosa), common two-banded seabream (Diplodus vulgaris) and bogue (Boops boops) catching efficiency. In the sea bass line fishing, catching efficiency was compared to between live fishing baits as annular seabream (Diplodus annularis) and grey mullet (Mugil cephalus), dead baits picarel (Spicara sp.) and cuttlefish (Sepia elegans) by Soykan \& Kınacigil (2013). Ateşşahin et al. (2015) determined the relationship between spinner hook sizes (2,3 and 4) and hook selectivity for rainbow trout (Oncorhynchus mykiss), a species important to recreational fisheries in Karakaya Dam Lake in Eastern Turkey.

Despite the fact that mud shrimp (Upogebia pusilla) is commonly used in handline fishing in Turkey, there are no studies conducted on its catching performance or efficiency, and there is only one study in the international literature (Erzini et al. 1998). 
To take into consideration cost disadvantages and not the availability of natural baits, fishers have started to look for alternative handline bait. In Bodrum region, anglers who practice amateur handline and commercial long line fishing have started to use the bait that is a mixture of pellet bait (ready-made bait to feed fishes in farms) and silicone (adhesive construction material known for its waterproofness). The mixture bait of silicon and pellet is only used in Turkey and there is no study examined the impact of the bait on catching performance.

In this study, catching performance and efficiency of three different bait types; live mud shrimp (natural), silicon pellet bait and siliconized mud shrimp bait, which was developed in this study, were investigated.

\section{Material and Methods}

Fishing experiments were carried out at monthly sampling periods between May 2016 and April 2017 in Zeytinlikuyu site, which located in Kıyıkışlacık Village of Muğla province, Milas. Catching activities were performed with groups of 3 or 4 people in the morning (beginning of the sunrise and +3 hours) and evening around (sunset -3 and sunset hours). The water depth of the sampling areas varied between 7 and 52 meters and the average depth was 22 meters. In the study bait handline which is named "gadide" in the region was used. The main body of the handline is $\varnothing 0.50 \mathrm{~mm}$, leader and snoods are $\varnothing 0.27-0.30 \mathrm{~mm}$, the snood length and distance are $10 \mathrm{~cm}$ and $20 \mathrm{~cm}$, respectively. Each set has 3 hooks and the hook is 4 no straight type. All hook sets used in the study were made as identical (Figure 1). The order of the baits was changed for each use.

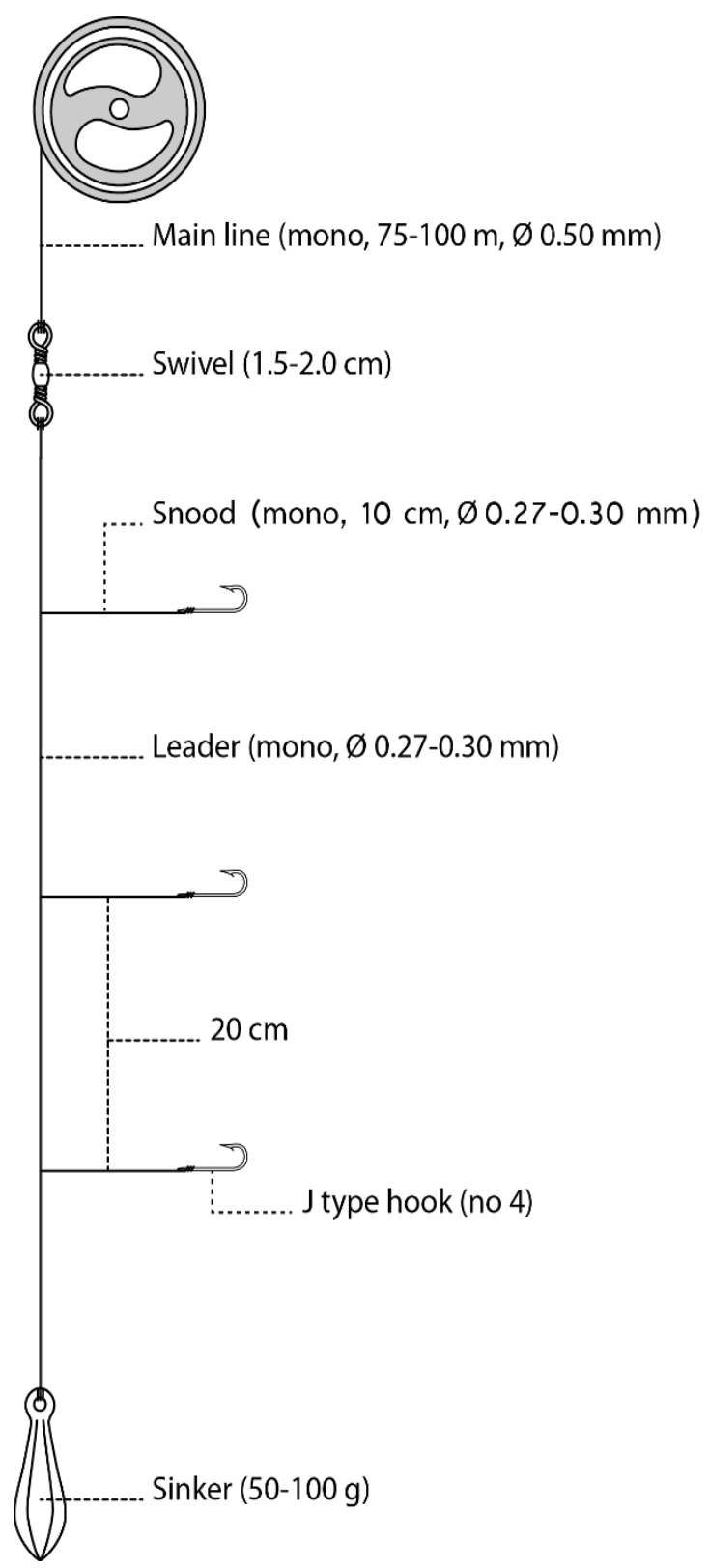

Figure 1- Handline used in experiments 
Three different baits were examined in the study;

I- Live (natural) mud shrimp; it was provided via purchasing from the producers. It was kept in cold storage during the fishing period and using as a live.

II- Siliconized mud shrimp; the mud shrimps which were provided by purchasing from producers were put down in a freezer for preventing the pain as much as possible based on the scientific ethical principles. After this process, the bodies of the mud shrimps were pressed with silicone. In this process, syringes filled with transparent aquarium silicone were used. The syringe was injected from the point where the abdomen was close to uropod directly to the mud shrimp (Figure 2) and kept at room temperature.

III- Silicon pellet; It is also sold in the fishing equipment market but not purchased in the study. Silicon pellet was made before fishing. The pellet using as feed was moisturized to soften for 10 hours. Afterwards, the bait embosses with a hammer or pulverized with a mixer, and then mixed with silicone, and re-filled in a silicon tube, and lastly, it was taken out of the silicon tube in the form of strips and allowed to dry. The next day, baits were cut in appropriate sizes for hooks used in the study (Figure $3)$.

Silicone, which is technically described as "polysiloxane", is used for wide range of purposes in different sectors such as automotive, electronics, beauty and self-care, food-beverage sector, construction and architecture. Transparent construction silicone aquarium silicone was preferred in the study due to their weak odour.

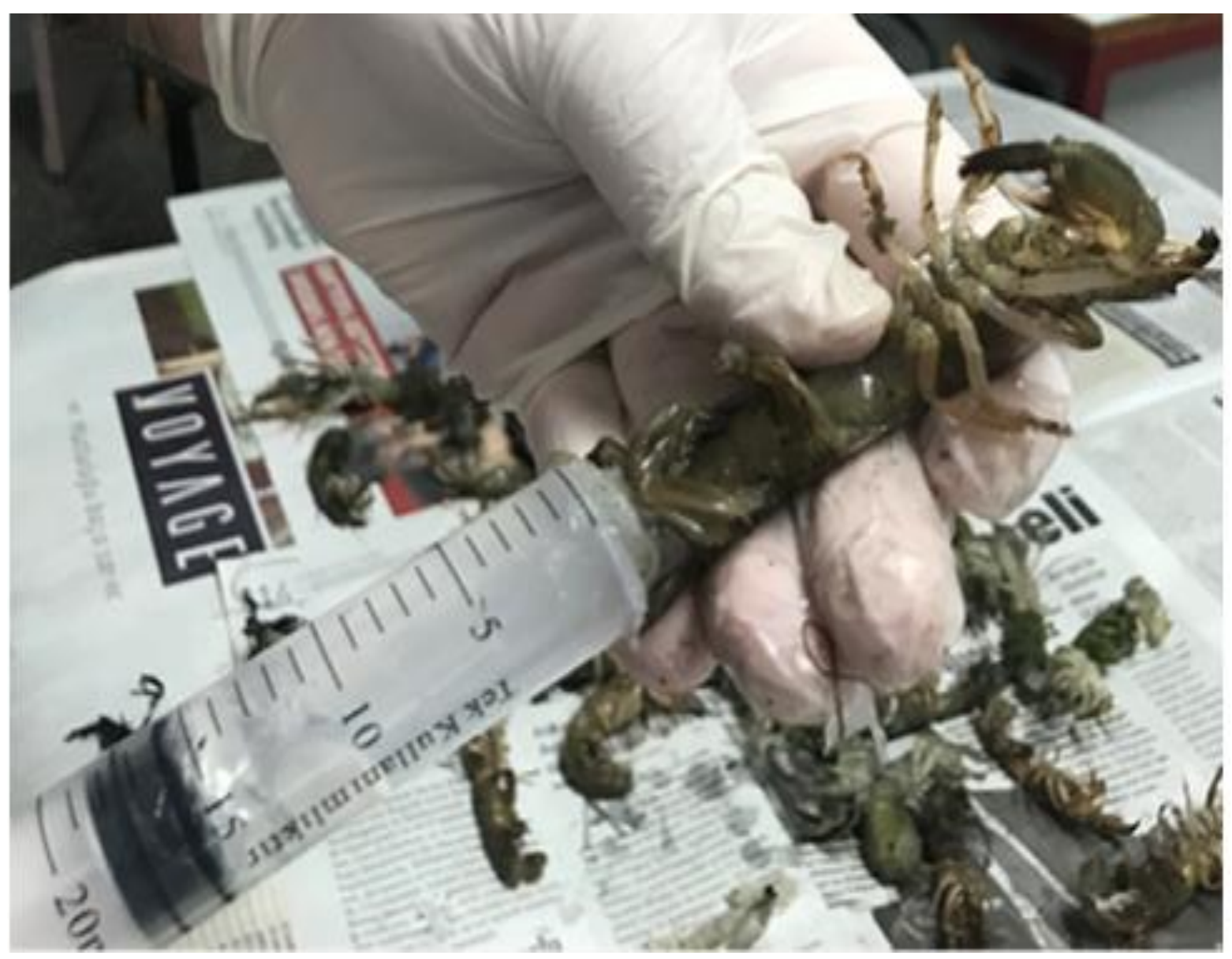

Figure 2- Siliconized of mud shrimp 


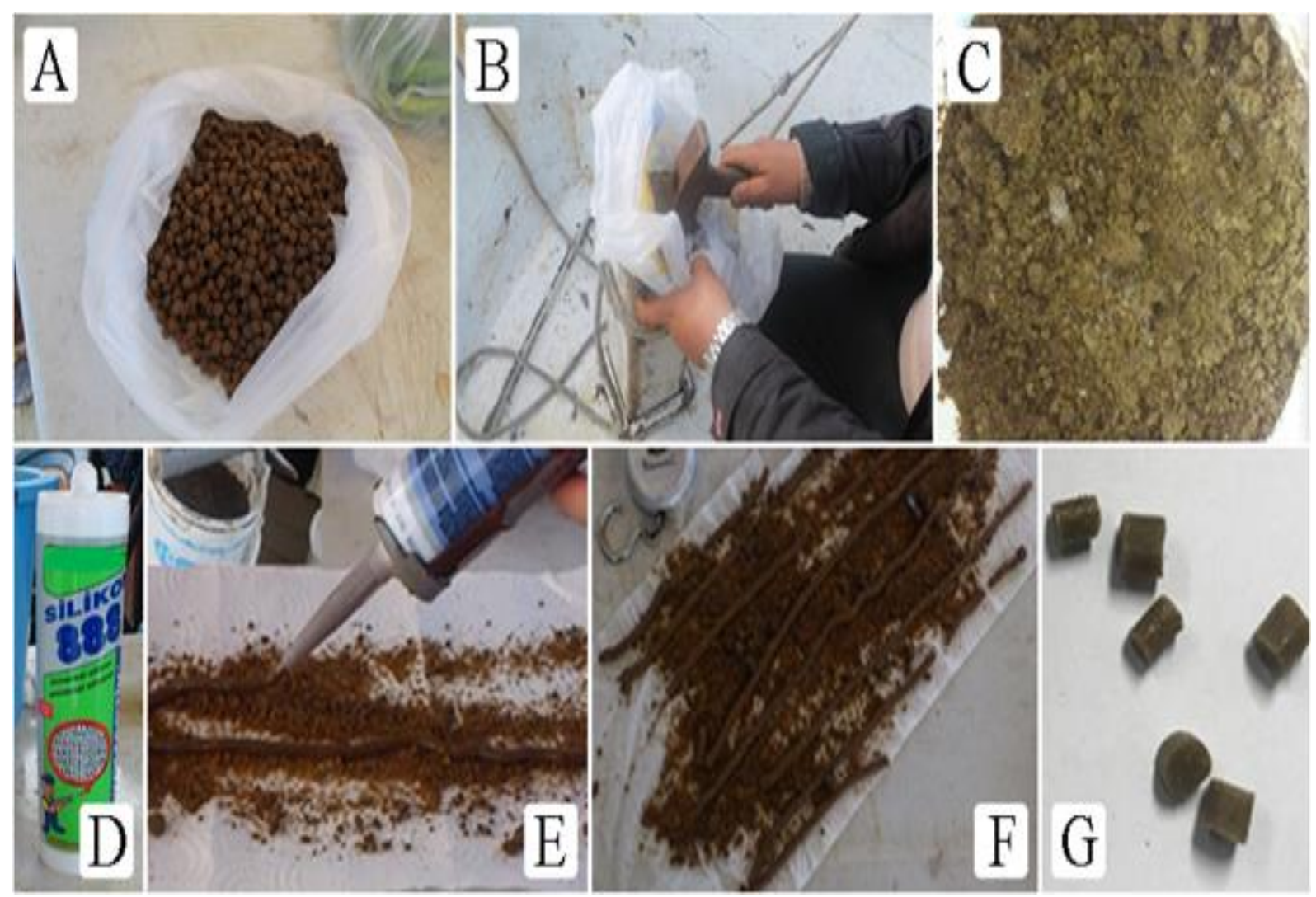

Figure 3- Making stages of the silicon pellet bait (A: Pellet feed, B: Pulverizing, C: Floured pellet, D: Transparent silicone, E: Silicone bait sticks, F: Drying, G: Silicon pellet bait that ready to fishing)

The individuals obtained from experiments were classified as live mud shrimp, silicon mud shrimp and silicon pellet. Each individual was weighed (nearest g) as and their total length (TL) was measured (nearest mm).

Before parametric tests were performed, the data were analyzed for homogeneity of variances and normal distribution using Levene and Kolmogorov-Smirnov one-sample test, respectively. If the homogeneity of variances and normal distribution were not confirmed, the data were not used for the test (Zar 1974).

The question of whether there is a statistical difference between the numbers of the sampled species in the $95 \%$ confidence interval or not was calculated with a $\chi^{2}$ test (chi-square) (Zar 1974);

$\chi^{2}=\sum \frac{(G-B)^{2}}{B}$

In the equation; $B$ presents the number of expected individuals and $G$ presents the number of observed individuals.

The question of whether there is a significant difference between the lengths of sampled individuals in the $95 \%$ interval was calculated by a Student's $t$-test (Zar 1974). The given equations were used in the cases that variances of the length groups were equal or non-equal;

$t=\frac{\bar{x}_{1}-\bar{x}_{2}}{\sqrt{\frac{S^{2}}{n}+\frac{S^{2}}{n}}} \quad t=\frac{\bar{x}_{1}-\bar{x}_{2}}{\sqrt{\frac{S_{1}^{2}}{n_{1}}+\frac{S_{2}^{2}}{n_{2}}}}$

In the equations, low index 1 and 2 represent independent groups, $\bar{x}$ represents the arithmetic mean and $S^{2}$ represents variance.

Whether there was a significant difference between the bait types used in the tests in the scope of the study and the mean length of the species in the $95 \%$ confidence interval or not was determined by using a one-way analysis of variance (ANOVA) (Zar 1974); 


\begin{tabular}{|c|c|c|c|c|}
\hline $\begin{array}{l}\text { Source of the } \\
\text { Variance }\end{array}$ & $S S$ & $d f$ & $M S$ & $F$ \\
\hline $\begin{array}{l}\text { Between } \\
\text { Groups }(B)\end{array}$ & $S S_{B}=\sum\left\lfloor\frac{\left(\sum x_{i}\right)^{2}}{n_{i}}\right\rfloor-\frac{\left(\sum x\right)^{2}}{N}$ & $d f_{B}=k-1$ & $M S_{B}=\frac{S S_{B}}{d f_{B}}$ & $F=\frac{M S_{B}}{M S_{W}}$ \\
\hline $\begin{array}{l}\text { Within a Group } \\
(W)\end{array}$ & $S S_{W}=S S_{T}-S S_{B}$ & $d f_{W}=N-k$ & $M S_{W}=\frac{S S_{W}}{d f_{W}}$ & \\
\hline Total $(T)$ & $S S_{T}=\sum x_{i}^{2}-\frac{\left(\sum x_{i}\right)^{2}}{N}$ & $d f_{T}=N-1$ & & \\
\hline
\end{tabular}

In the equations; $n_{i}$ represents the number of individuals in group $i, N$ represents the number of individuals in all groups, $\bar{x}_{i}$ represents the arithmetic means of the group $i$, k represents the total number of groups, SS represents sum the of squares, $d f$ represents the degree of freedom, MS represents mean square and F represents calculated criteria value.

The Catch per Unit Effort (CPUE) and Yield per Unit Effort (YPUE) were calculated respectively as; a number of individuals that can are catchability with one hook and the amount of the yielded catchability (gram) (Aydın, 2011);

$\mathrm{CPUE}=\frac{\sum n}{\sum h \times \sum t}$ and $\quad \mathrm{YPUE}=\frac{\sum W}{\sum h \times \sum t}$

In the equations, $n$ represents the number of individuals, $W$ represents the total weight of individuals (g), $h$ represents the number of hooks used in a set, and $t$ represents the duration of catching.

Mathematical (meteorological) seasons are used for the northern hemisphere for the seasonal sampling periods. According to this, March, April and May data are spring; June, July and August data are summer, September, October and November data are fall and December, January and February data are used as winter data set (TSMS 2017).

\section{Results and Discussion}

Results of the catching performance and efficiency of mud shrimp have been revealed for the first time in Turkey. Furthermore, the findings on catching efficiency and catching a performance of mud shrimp have been presented worldwide for the first time with this study. As far as it is known, the silicone pellet mixture is only used in Turkey and the results on its catching performance have been introduced for the first time.

A total of 590 individuals that belongs to 30 species were sampled in the fishing duration of 2427 minutes (40 hours and 27 minutes). These species included; 588 bony fishes (99.66\%), and 2 cephalopods $(0.34 \%)$. According to the results, the most frequently samples were (>5\%); annular seabream (Diplodus annularis, 23.90\%), common pandora (Pagellus erythrinus, $18.47 \%$ ), common two-banded seabream (Diplodus vulgaris, 15.93\%), gilthead seabream (Sparus aurata, 11.53\%) and brown comber (Serranus hepatus, 9.49\%) (Table 1). The first five species that were sampled in the study constituted $79.32 \%$ of the total catches.

In the study, 50.7\% of 590 individuals $(n=299)$ were sampled with live Mediterranean mud shrimp, and this bait was respectively followed by silicone Mediterranean mud shrimp $(n=264)$ with $44.7 \%$ and silicone pellet bait $(n=27)$ with $4.6 \%$.

The study results showed that the species caught with live Mediterranean mud shrimp most frequently were (\%>10); annular seabream with $24.41 \%$, common pandora $19.06 \%$, gilthead seabream $14.05 \%$, common two-banded seabream $13.71 \%$ and brown comber $11.04 \%$ (Table 2). Most frequently were (\%>5) species obtained siliconized mud shrimp were; annular seabream $22.35 \%$, common pandora $19.32 \%$, common two-banded seabream $17.42 \%$, gilthead seabream $9.85 \%$ and brown cumber $7.95 \%$. Lastly, the species which were caught with silicone pellet bait were (\%>10); annular sea bream 33.33\%, common two-banded seabream $25.93 \%$ and gilthead seabream $11.11 \%$. 
Table 1- Experimented species, numbers, percentage distribution with lengths and weights distribution, arithmetic mean and standard error Bony fishes

\begin{tabular}{|c|c|c|c|c|c|c|c|c|c|c|c|c|}
\hline Family & Species name & Common name & $n$ & $\%$ & $L_{\min }$ & $L_{\max }$ & $L_{\text {mean }}$ & $L_{\mathrm{se}}$ & $W_{\min }$ & $W_{\max }$ & $W_{\text {mean }}$ & $W_{\text {se }}$ \\
\hline Balistidae & Balistes capriscus & Grey triggerfish & 5 & 0.85 & 17.5 & 32.5 & 25.46 & 2.94 & 94.0 & 617.2 & 351.33 & 103.55 \\
\hline Caranginae & $\begin{array}{l}\text { Trachurus } \\
\text { mediterraneus }\end{array}$ & $\begin{array}{l}\text { Mediterranean } \\
\text { horse mackerel }\end{array}$ & 3 & 0.51 & 14.6 & 24.2 & 18.37 & 2.96 & 26.5 & 130.0 & 64.90 & 32.73 \\
\hline Engraulidae & Engraulis encrasicolus & European anchovy & 1 & 0.17 & 10.5 & 10.5 & 10.50 & & 8.1 & 8.1 & 8.10 & \\
\hline Gobiidae & Gobius niger & Black goby & 11 & 1.86 & 8.0 & 12.6 & 10.52 & 0.41 & 6.0 & 21.6 & 15.15 & 1.53 \\
\hline Labridae & Labrus viridis & Green wrasse & 1 & 0.17 & 21.5 & 21.5 & 21.50 & & 101.0 & 101.0 & 101.00 & \\
\hline Moronidae & Dicentrarchus labrax & European seabass & 1 & 0.17 & 36.0 & 36.0 & 36.00 & & 459.0 & 459.0 & 459.00 & \\
\hline Mullidae & $\begin{array}{l}\text { Mullus barbatus } \\
\text { barbatus }\end{array}$ & Red mullet & 1 & 0.17 & 13.0 & 13.0 & 13.00 & & 24.0 & 24.0 & 23.98 & \\
\hline Sciaenidae & Umbrina cirrosa & Shi drum & 1 & 0.17 & 25.5 & 25.5 & 25.50 & & 193.0 & 193.0 & 193.00 & \\
\hline Scombridae & Scomber japonicus & Chub mackerel & 4 & 0.68 & 28.5 & 33.5 & 30.53 & 1.09 & 175.1 & 310.8 & 239.00 & 27.87 \\
\hline Scorpaenidae & Scorpaena scrofa & Red scorpionfish & 1 & 0.17 & 14.5 & 14.5 & 14.50 & & 54.1 & 54.1 & 54.10 & \\
\hline \multirow{3}{*}{ Serranidae } & Serranus cabrilla & Comber & 21 & 3.56 & 7.4 & 18.2 & 12.06 & 0.75 & 7.1 & 85.0 & 29.97 & 4.70 \\
\hline & Serranus hepatus & Brown comber & 56 & 9.49 & 7.2 & 25.1 & 9.32 & 0.31 & 6.5 & 255.7 & 19.29 & 4.38 \\
\hline & Serranus scriba & Painted comber & 6 & 1.02 & 12.5 & 19.4 & 15.37 & 1.03 & 26.5 & 106.2 & 58.62 & 11.83 \\
\hline Siganidae & Siganus rivulatus & Marbled spinefoot & 1 & 0.17 & 13.5 & 13.5 & 13.50 & & 75.0 & 75.0 & 75.00 & \\
\hline \multirow{12}{*}{ Sparidae } & Boops boops & Bogue & 14 & 2.37 & 8.4 & 18.6 & 13.71 & 0.58 & 5.9 & 62.1 & 32.64 & 3.73 \\
\hline & Dentex maroccanus & Morocco dentex & 2 & 0.34 & 22.1 & 26.1 & 24.10 & 2.00 & 144.0 & 206.0 & 175.00 & 31.00 \\
\hline & Diplodus annularis & Annular seabream & 141 & 23.90 & 8.5 & 17.8 & 14.00 & 0.14 & 10.0 & 110.0 & 50.45 & 1.61 \\
\hline & Diplodus puntazzo & $\begin{array}{l}\text { Sharpsnout } \\
\text { seabream }\end{array}$ & 1 & 0.17 & 23.8 & 23.8 & 23.80 & & 260.0 & 260.0 & 260.00 & \\
\hline & Diplodus vulgaris & $\begin{array}{l}\text { Common two- } \\
\text { banded seabream }\end{array}$ & 94 & 15.93 & 8.9 & 26.5 & 16.86 & 0.45 & 11.9 & 327.3 & 96.15 & 6.69 \\
\hline & $\begin{array}{l}\text { Lithognathus } \\
\text { mormyrus }\end{array}$ & Sand steenbras & 6 & 1.02 & 20.6 & 24.6 & 22.55 & 0.56 & 113.4 & 163.9 & 144.82 & 7.44 \\
\hline & Oblada melanura & Saddled seabream & 2 & 0.34 & 18.0 & 19.0 & 18.50 & 0.50 & 69.0 & 80.6 & 74.80 & 5.80 \\
\hline & Pagellus acarne & Axillary seabream & 2 & 0.34 & 12.0 & 12.8 & 12.40 & 0.40 & 21.9 & 23.2 & 22.55 & 0.65 \\
\hline & Pagellus erythrinus & Common pandora & 109 & 18.47 & 10.0 & 31.5 & 20.19 & 0.49 & 12.0 & 392.0 & 125.45 & 8.52 \\
\hline & Pagrus caeruleostictus & $\begin{array}{l}\text { Bluespotted } \\
\text { seabream }\end{array}$ & 4 & 0.68 & 7.9 & 27.5 & 22.05 & 4.74 & 229.0 & 310.0 & 272.24 & 16.74 \\
\hline & Sparus aurata & Gilthead seabream & 68 & 11.53 & 13.0 & 23.6 & 17.52 & 0.33 & 5.0 & 450.0 & 83.74 & 7.33 \\
\hline & Spicara maena & Blotched picarel & 24 & 4.07 & 10.9 & 16.7 & 14.47 & 0.26 & 14.7 & 52.5 & 35.66 & 1.88 \\
\hline Tetraodontidae & $\begin{array}{l}\text { Lagocephalus } \\
\text { spadiceus }\end{array}$ & $\begin{array}{l}\text { Half-smooth } \\
\text { golden pufferfish }\end{array}$ & 6 & 1.02 & 23.2 & 29.4 & 25.92 & 1.01 & 230.0 & 510.0 & 352.50 & 46.15 \\
\hline \multirow[t]{2}{*}{ Trachinidae } & Trachinus draco & Greater weever & 2 & 0.34 & 23.4 & 24.9 & 24.15 & 0.75 & 85.0 & 123.0 & 104.00 & 19.00 \\
\hline & & Sum of bony fishes: & 588 & 99.66 & & & & & & & & \\
\hline Loliginidae & $\begin{array}{l}\text { Loligo } \\
\text { vulgaris }\end{array}$ & $\begin{array}{l}\text { European } \\
\text { squid }\end{array}$ & 1 & 0.17 & 23.2 & 23.2 & 23.20 & & 310.0 & 310.0 & 310.00 & \\
\hline \multirow[t]{2}{*}{ Octopodidae } & $\begin{array}{l}\text { Octopus } \\
\text { vulgaris }\end{array}$ & $\begin{array}{l}\text { Common } \\
\text { octopus }\end{array}$ & 1 & 0.17 & & & & & 2680.0 & 2680.0 & 2680.0 & \\
\hline & & Sum of cephalopods: & 2 & 0.34 & & & & & & & & \\
\hline Grand total: & & & 590 & 100.00 & & & & & & & & \\
\hline
\end{tabular}

$n$, Sample size; $\%$, Ratio in total; $L$, Total length $(\mathrm{cm}) ; M L$, Mantle length (cm); $W$, Total weight (gr). min, max, mean and se: Minimum, maximum, mean and standard error. Species listed in alphabetical order according to family and species name. Scientific and common names are based on FishBase (Froese $\&$ Pauly 2019) and SeaLifeBase (Palomares \& Pauly 2019). 
Aydın \& Illkyaz - Journal of Agricultural Sciences (Tarım Bilimleri Dergisi), 2021, 27(1):219-230

Table 2- Experimented species with bait types used in the study, numbers and rates with result of statistical tests

\begin{tabular}{|c|c|c|c|c|c|c|c|c|c|c|c|c|c|c|}
\hline \multirow{2}{*}{ Species name } & \multicolumn{4}{|c|}{$L M$} & \multicolumn{3}{|c|}{$S M$} & \multicolumn{3}{|c|}{$S P$} & \multicolumn{4}{|c|}{$P$} \\
\hline & $\Sigma n$ & $n$ & $\% T$ & $\% G$ & $n$ & $\% T$ & $\% G$ & $n$ & $\% T$ & $\% G$ & $T$ & $L M-S M$ & $L M-S P$ & $S M-S P$ \\
\hline Balistes capriscus & 5 & 1 & 20.0 & 0.3 & 3 & 60.0 & 1.1 & 1 & 20.0 & 3.7 & - & - & - & - \\
\hline Trachurus mediterraneus & 3 & 0 & 0.0 & 0.0 & 3 & 100.0 & 1.1 & 0 & 0.0 & 0.0 & + & - & $x$ & - \\
\hline Engraulis encrasicolus & 1 & 0 & 0.0 & 0.0 & 1 & 100.0 & 0.4 & 0 & 0.0 & 0.0 & - & - & $x$ & - \\
\hline Gobius niger & 11 & 3 & 27.3 & 1.0 & 7 & 63.6 & 2.7 & 1 & 9.1 & 3.7 & - & - & - & + \\
\hline Labrus viridis & 1 & 0 & 0.0 & 0.0 & 1 & 100.0 & 0.4 & 0 & 0.0 & 0.0 & - & - & $x$ & - \\
\hline Dicentrarchus labrax & 1 & 0 & 0.0 & 0.0 & 1 & 100.0 & 0.4 & 0 & 0.0 & 0.0 & - & - & $\times$ & - \\
\hline Mullus barbatus barbatus & 1 & 0 & 0.0 & 0.0 & 1 & 100.0 & 0.4 & 0 & 0.0 & 0.0 & - & - & $x$ & - \\
\hline Umbrina cirrosa & 1 & 1 & 100.0 & 0.3 & 0 & 0.0 & 0.0 & 0 & 0.0 & 0.0 & - & - & - & $x$ \\
\hline Scomber japonicus & 4 & 2 & 50.0 & 0.7 & 1 & 25.0 & 0.4 & 1 & 25.0 & 3.7 & - & - & - & - \\
\hline Scorpaena scrofa & 1 & 0 & 0.0 & 0.0 & 1 & 100.0 & 0.4 & 0 & 0.0 & 0.0 & - & - & $x$ & - \\
\hline Serranus cabrilla & 21 & 10 & 47.6 & 3.3 & 11 & 52.4 & 4.2 & 0 & 0.0 & 0.0 & + & - & + & + \\
\hline Serranus hepatus & 56 & 33 & 58.9 & 11.0 & 21 & 37.5 & 8.0 & 2 & 3.6 & 7.4 & + & - & + & + \\
\hline Serranus scriba & 6 & 4 & 66.7 & 1.3 & 2 & 33.3 & 0.8 & 0 & 0.0 & 0.0 & - & - & + & - \\
\hline Siganus rivulatus & 1 & 1 & 100.0 & 0.3 & 0 & 0.0 & 0.0 & 0 & 0.0 & 0.0 & - & - & - & $\times$ \\
\hline Boops boops & 14 & 7 & 50.0 & 2.3 & 5 & 35.7 & 1.9 & 2 & 14.3 & 7.4 & - & - & - & - \\
\hline Dentex maroccanus & 2 & 2 & 100.0 & 0.7 & 0 & 0.0 & 0.0 & 0 & 0.0 & 0.0 & - & - & - & $x$ \\
\hline Diplodus annularis & 141 & 73 & 51.8 & 24.4 & 59 & 41.8 & 22.4 & 9 & 6.4 & 33.3 & + & - & + & + \\
\hline Diplodus puntazzo & 1 & 0 & 0.0 & 0.0 & 1 & 100.0 & 0.4 & 0 & 0.0 & 0.0 & - & - & $x$ & - \\
\hline Diplodus vulgaris & 94 & 41 & 43.6 & 13.7 & 46 & 48.9 & 17.4 & 7 & 7.4 & 25.9 & + & - & + & + \\
\hline Lithognathus mormyrus & 6 & 2 & 33.3 & 0.7 & 4 & 66.7 & 1.5 & 0 & 0.0 & 0.0 & - & - & - & + \\
\hline Oblada melanura & 2 & 0 & 0.0 & 0.0 & 2 & 100.0 & 0.8 & 0 & 0.0 & 0.0 & - & - & $x$ & - \\
\hline Pagrus caeruleostictus & 4 & 1 & 25.0 & 0.3 & 3 & 75.0 & 1.1 & 0 & 0.0 & 0.0 & - & - & - & - \\
\hline Sparus aurata & 68 & 42 & 61.8 & 14.1 & 26 & 38.2 & 9.9 & 0 & 0.0 & 0.0 & + & - & + & + \\
\hline Spicara maena & 24 & 13 & 54.2 & 4.4 & 8 & 33.3 & 3.0 & 3 & 12.5 & 11.1 & + & - & + & - \\
\hline Lagocephalus spadiceus & 6 & 4 & 66.7 & 1.3 & 2 & 33.3 & 0.8 & 0 & 0.0 & 0.0 & - & - & + & - \\
\hline Trachinus draco & 2 & 0 & 0.0 & 0.0 & 2 & 100.0 & 0.8 & 0 & 0.0 & 0.0 & - & - & $x$ & - \\
\hline Loligo vulgaris & 1 & 1 & 100.0 & 0.3 & 0 & 0.0 & 0.0 & 0 & 0.0 & 0.0 & - & - & - & $\times$ \\
\hline Octopus vulgaris & 1 & 0 & 0.0 & 0.0 & 1 & 100.0 & 0.4 & 0 & 0.0 & 0.0 & - & - & $x$ & - \\
\hline Total: & 590 & 299 & 50.7 & 100.0 & 264.0 & 1741.5 & 100.0 & 27.0 & 99.2 & 100.0 & + & - & + & + \\
\hline
\end{tabular}

LM, Live Mediterranean mud shrimp; SM, Silicone Mediterranean mud shrimp; SP, Silicone pellet bait; $\Sigma$, Total. $n$ : Sample size; $\%$ T, Ratio in species total; $\% \mathrm{G}$, Ratio in group total; $P$, Chi-square $\left(\chi^{2}\right)$ test results of $95 \%$ confidence limit; + , Statistically difference. - , Statistically no difference; $\times$, Insufficient data for testing; $\mathrm{T}$, Test results of the all bait types

The study findings showed that among the species caught with different three different baits were statistically different $\left(\chi^{2}\right.$, $\mathrm{P}<0.05)$.

According to the results, no statistically significant difference was found between the sample sizes of other species and bait types $\left(\chi^{2}, \mathrm{P}>0.05\right)$. Moreover, when the total number of caught individuals was taken into the consideration, the number of individuals caught by all three types of bait was statistically different than each other $\left(\chi^{2}, \mathrm{P}<0.05\right)$. In terms of the number of individuals, no difference was found between live Mediterranean mud shrimp and silicone Mediterranean mud shrimp baits in the paired comparison $\left(\chi^{2}, \mathrm{P}>0.05\right)$. On the other hand, a difference was found between live Mediterranean mud shrimp-silicone pellet and also between silicone Mediterranean mud shrimp and silicone pellet $\left(\chi^{2}, \mathrm{P}<0.05\right)($ Table 2$)$.

In the study, the smallest-size of an individual was $7.2 \mathrm{~cm}$ with brown comber (Serranus hepatus) and the biggest one was $36 \mathrm{~cm}$ TL with European seabass (Dicentrarchus labrax). The mean lengths of the samples that caught with different baits were statistics different for grey triggerfish, black goby, chub mackerel, brown comber, comber, bogue, annular seabream, common Pandora and blotched picarel $(\mathrm{P}<0.05)$, however, it was not statistically different for common two-banded seabream $($ ANOVA, 
$\mathrm{P}>0.05)$. In the comparison between live Mediterranean mud shrimp and silicone Mediterranean mud shrimp, no difference was found for the mean lengths of all species (ANOVA, P>0.05). In the comparison made between paired bait groups, the difference was found that silicon Mediterranean mud shrimp-silicone pellet bait and live Mediterranean mud shrimp-silicone pellet bait for common two-banded seabream (ANOVA, P<0.05). Due to the insufficient data, test statistics were not applied for the 20 species (apart from the aforementioned ones) (Table 3).

Table 3- Lengths ranges, mean lengths, standard error and result of statistical test differences of experimented species with bait types used in the study

\begin{tabular}{|c|c|c|c|c|c|c|c|c|c|c|c|c|c|c|c|c|c|c|c|}
\hline & \multicolumn{5}{|c|}{ Live Med. mud shrimp } & \multicolumn{6}{|c|}{ Silicone Med. mud shrimp } & \multicolumn{4}{|c|}{ Silicone pellet bait } & \multicolumn{4}{|c|}{$P$} \\
\hline Species name & $n$ & $L_{m i n}$ & $L_{m a k}$ & $L_{o r t}$ & $L_{s e}$ & $n$ & $L_{\min }$ & $L_{m a k}$ & $L_{\text {ort }}$ & $L_{s e}$ & $n$ & $L_{\min }$ & $L_{m a k}$ & $L_{o r t}$ & $L_{s e}$ & $T$ & $\begin{array}{l}L M- \\
S M\end{array}$ & $\begin{array}{l}L M- \\
S P\end{array}$ & $S M-S P$ \\
\hline $\begin{array}{l}\text { Balistes } \\
\text { capriscus }\end{array}$ & 1 & 32.5 & 32.5 & 32.50 & & 3 & 17.5 & 32.0 & 23.77 & 4.30 & 1 & 23.5 & 23.5 & 23.50 & & - & - & - & - \\
\hline $\begin{array}{l}\text { Trachurus } \\
\text { mediterraneus }\end{array}$ & & & & & & 3 & 14.6 & 24.2 & 18.37 & 2.96 & & & & & & & & & \\
\hline $\begin{array}{l}\text { Engraulis } \\
\text { encrasicolus }\end{array}$ & & & & & & 1 & 10.5 & 10.5 & 10.50 & & & & & & & & & & \\
\hline Gobius niger & 3 & 9.9 & 12.6 & 11.53 & 0.83 & 7 & 8.0 & 11.8 & 10.03 & 0.46 & 1 & 10.9 & 10.9 & 10.90 & & - & - & - & - \\
\hline $\begin{array}{l}\text { Labrus } \\
\text { viridis }\end{array}$ & & & & & & 1 & 21.5 & 21.5 & 21.50 & & & & & & & & & & \\
\hline $\begin{array}{l}\text { Dicentrarchus } \\
\text { labrax }\end{array}$ & & & & & & 1 & 36.0 & 36.0 & 36.00 & & & & & & & & & & \\
\hline $\begin{array}{l}\text { Mullus barbatus } \\
\text { barbatus }\end{array}$ & & & & & & 1 & 13.0 & 13.0 & 13.00 & & & & & & & & & & \\
\hline Umbrina cirrosa & 1 & 25.5 & 25.5 & 25.50 & & & & & & & & & & & & & & & \\
\hline $\begin{array}{l}\text { Scomber } \\
\text { japonicus }\end{array}$ & 2 & 30.7 & 33.5 & 32.10 & 1.40 & 1 & 29.4 & 29.4 & 29.40 & & 1 & 28.5 & 28.5 & 28.50 & & - & - & - & $x$ \\
\hline $\begin{array}{l}\text { Scorpaena } \\
\text { scrofa }\end{array}$ & & & & & & 1 & 14.5 & 14.5 & 14.50 & & & & & & & & & & \\
\hline $\begin{array}{l}\text { Serranus } \\
\text { cabrilla }\end{array}$ & 10 & 7.4 & 16.0 & 11.17 & 0.92 & 11 & 8.0 & 18.2 & 12.86 & 1.14 & & & & & & & - & & \\
\hline $\begin{array}{l}\text { Serranus } \\
\text { hepatus } \\
\end{array}$ & 33 & 7.2 & 25.1 & 9.55 & 0.50 & 21 & 7.3 & 10.3 & 8.97 & 0.18 & 2 & 8.6 & 9.5 & 9.05 & 0.45 & - & - & - & - \\
\hline Serranus scriba & 4 & 12.5 & 19.4 & 15.48 & 1.43 & 2 & 13.3 & 17.0 & 15.15 & 1.85 & & & & & & & - & & \\
\hline $\begin{array}{l}\text { Siganus } \\
\text { rivulatus }\end{array}$ & 1 & 13.5 & 13.5 & 13.50 & & & & & & & & & & & & & & & \\
\hline Boops boops & 7 & 8.4 & 18.6 & 13.66 & 1.14 & 5 & 12.6 & 15.4 & 13.72 & 0.50 & 2 & 12.9 & 14.9 & 13.90 & 1.00 & - & - & - & - \\
\hline $\begin{array}{l}\text { Dentex } \\
\text { maroccanus }\end{array}$ & 2 & 22.1 & 26.1 & 24.10 & 2.00 & & & & & & & & & & & & & & \\
\hline $\begin{array}{l}\text { Diplodus } \\
\text { annularis }\end{array}$ & 73 & 9.0 & 17.0 & 14.11 & 0.18 & 59 & 8.5 & 17.4 & 13.78 & 0.24 & 9 & 11.4 & 17.8 & 14.47 & 0.65 & - & - & - & - \\
\hline $\begin{array}{l}\text { Diplodus } \\
\text { puntazzo }\end{array}$ & & & & & & 1 & 23.8 & 23.8 & 23.80 & & & & & & & & & & \\
\hline $\begin{array}{l}\text { Diplodus } \\
\text { vulgaris }\end{array}$ & 41 & 9.0 & 24.1 & 16.68 & 0.69 & 46 & 9.2 & 26.5 & 17.67 & 0.60 & 7 & 8.9 & 19.0 & 12.61 & 1.30 & + & - & + & + \\
\hline $\begin{array}{l}\text { Lithognathus } \\
\text { mormyrus }\end{array}$ & 2 & 21.9 & 23.4 & 22.65 & 0.75 & 4 & 20.6 & 24.6 & 22.50 & 0.82 & & & & & & & - & & \\
\hline $\begin{array}{l}\text { Oblada } \\
\text { melanura }\end{array}$ & & & & & & 2 & 18.0 & 19.0 & 18.50 & 0.50 & & & & & & & & & \\
\hline Pagellus acarne & 1 & 12.8 & 12.8 & 12.80 & & 1 & 12.0 & 12.0 & 12.00 & & & & & & & & $x$ & & \\
\hline $\begin{array}{l}\text { Pagellus } \\
\text { erythrinus }\end{array}$ & 57 & 10.7 & 30.2 & 19.14 & 0.59 & 51 & 10.0 & 31.5 & 21.35 & 0.79 & 1 & 20.9 & 20.9 & 20.90 & & - & - & - & - \\
\hline $\begin{array}{l}\text { Pagrus } \\
\text { caeruleostictus }\end{array}$ & 1 & 27.2 & 27.2 & 27.20 & & 3 & 7.9 & 27.5 & 20.33 & 6.24 & & & & & & & - & & \\
\hline Sparus aurata & 42 & 13.0 & 23.6 & 17.17 & 0.43 & 26 & 14.7 & 23.3 & 18.07 & 0.51 & & & & & & & - & & \\
\hline Spicara maena & 13 & 13.7 & 16.7 & 14.97 & 0.21 & 8 & 12.3 & 15.4 & 13.98 & 0.45 & 3 & 10.9 & 15.2 & 13.60 & 1.36 & - & - & - & - \\
\hline $\begin{array}{l}\text { Lagocephalus } \\
\text { spadiceus }\end{array}$ & 4 & 23.2 & 29.4 & 26.20 & 1.51 & 2 & 24.2 & 26.5 & 25.35 & 1.15 & & & & & & & - & & \\
\hline Trachinus draco & & & & & & 2 & 23.4 & 24.9 & 24.15 & 0.75 & & & & & & & & & \\
\hline Loligo vulgaris ${ }^{*}$ & 1 & 23.2 & 23.2 & 23.20 & & & & & & & & & & & & & & & \\
\hline $\begin{array}{l}\text { Octopus } \\
\text { vulgaris }^{* *}\end{array}$ & & & & & & 1 & 2.68 & 2.68 & 2.68 & & & & & & & & & & \\
\hline Total: & 299 & & & & & 26 & & & & & & & & & & & & & \\
\hline
\end{tabular}

$n$, Sample size. $L$ : Total length $(\mathrm{cm})$; *, Mantle length $(\mathrm{cm})$; **, Total weight $(\mathrm{kg})$. min, max, mean and se: Minimum, maximum, mean and standard error; $P$, One way ANOVA test results of $95 \%$ confidence limit; +, Statistically difference; - , Statistically no difference; $\times$, Insufficient data for testing; LM, Live Mediterranean mud shrimp; SM, Silicone Mediterranean mud shrimp; SP, Silicone pellet bait

CPUE value was calculated as $1.03 \mathrm{~h} / \mathrm{h}$ and YPUE value was calculated as $89.06 \mathrm{~g} / \mathrm{h}$. According to the seasonal alterations, the most productive season was found as autumn (CPUE; $1.99 \mathrm{n} / \mathrm{h}$, YPUE: $180.18 \mathrm{~g} / \mathrm{h}$ ) in spite of this the least productive season 
was spring (CPUE; $0.28 \mathrm{n} / \mathrm{h}$, YPUE: $24.63 \mathrm{~g} / \mathrm{h}$ ). For the summer and winter seasons, the CPUE values were respectively found as 0.89 and $1.45 \mathrm{n} / \mathrm{h}$; additionally, the YPUE values were found as 71.77 and $124.92 \mathrm{~g} / \mathrm{h}$ (Figure 4).

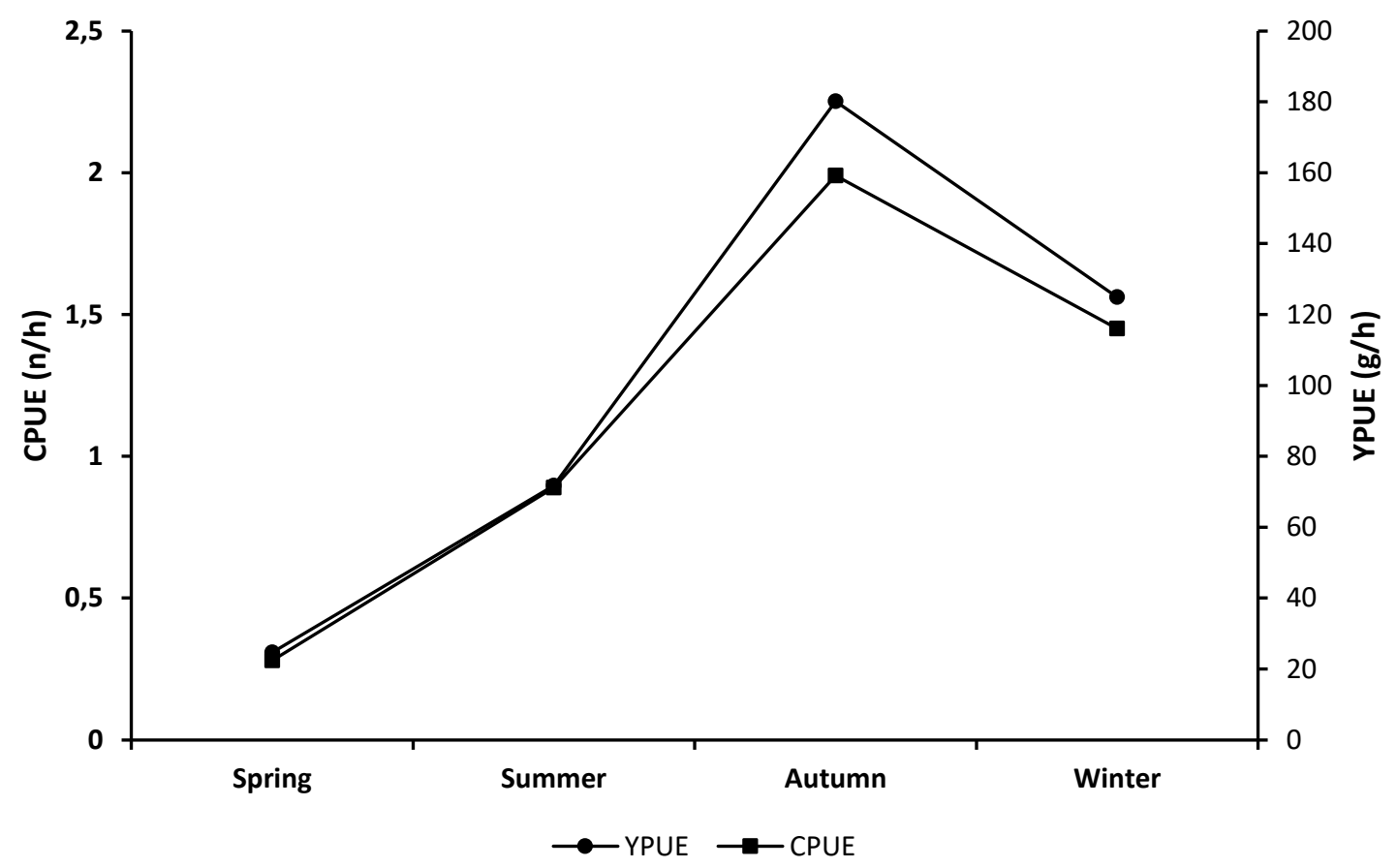

Figure 4- Seasonal changes of CPUE and YPUE values

The CPUE value of handline fishing practised with live Mediterranean mud shrimp was calculated as $1.57,1.38$ for silicone Mediterranean mud shrimp and $0.14 \mathrm{n} / \mathrm{h}$ for silicone pellet bait. On the other hand, the YPUE value of handline fishing was found as 121.84 for live Mediterranean mud shrimp and 137.73 for silicone Mediterranean mud shrimp and $7.62 \mathrm{~g} / \mathrm{h}$ for silicone pellet bait (Figure 5).

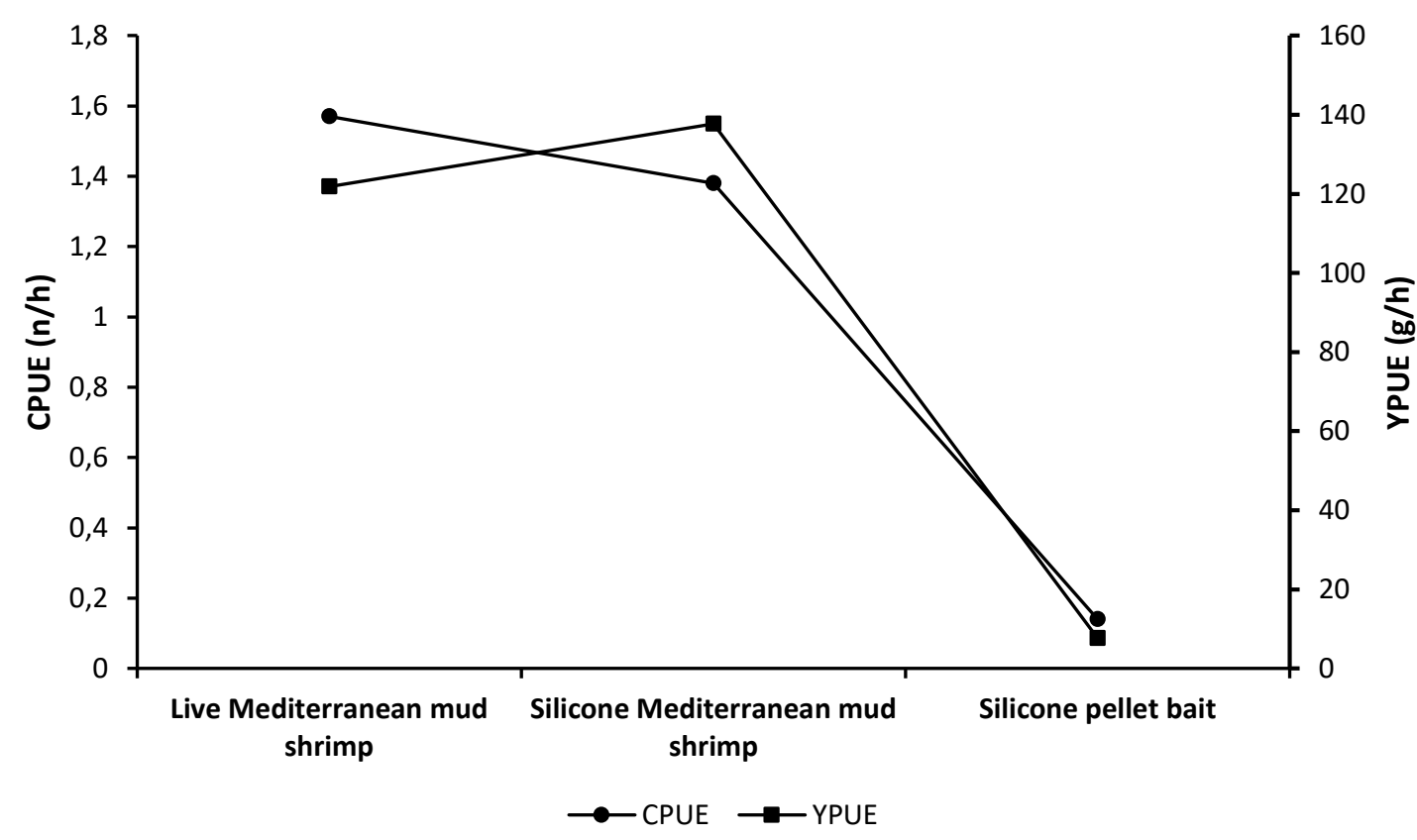

Figure 5- CPUE and YPUE values for bait types

The study findings revealed that the least productive season for all bait types was spring according to the seasonal productivity data of all three types of baits tested in the study in terms of handline fishing. The results showed that autumn was the most productive season for live Mediterranean mud shrimp and silicone Mediterranean mud shrimp. However, for silicone pellet bait, the most productive season was winter. 
The numbers of individuals, which were caught with live mud shrimp and silicone mud shrimp, were relatively approximate to each other (50.7 and 44.7\%, respectively). This result shows that silicone mud shrimp bait can be considered as an alternative to the live mud shrimp bait. Among the caught species, brown comber, seabream, two banded bream and common pandora have high catching rate for all types of baits. On the other hand, it was observed that mud shrimp which was used as a live bait died in the catching activities and lost the water it contains, and therefore its catching efficiency has tended to decrease. Løkkeborg (1991), minced raw materials as feeding stimulants and nylon bag as reinforcement was tested in fishing trials for cusk (Brosme brosme), ling (Molva molva), Atlantic cod (Gadus morhua) and haddock (Melanogrammus aeglefinus). According to study results, compared with natural bait, minced Atlantic herring (Clupea harengus) enclosed in a nylon bag gave a higher catch rate for haddock (58\%), torsk and ling. The texture of the nylon bag had a negative effect on the catch rate, most pronounced for cod and haddock. Interviews conducted with the fishers in the study area demonstrated that silicone pellet catches more products in certain periods (dark of the moon) and catch certain species more (seabass, mackerel etc.). Therefore, the efficiency of silicone pellet should be investigated in another study.

The seasonal comparison of CPUE and YPUE values showed that fall is the most productive season (CPUE; $1.99 n / h$, YPUE: $180.18 \mathrm{~g} / \mathrm{h}$ ) followed by winter (CPUE; $1.45 \mathrm{n} / \mathrm{h}$ and $124.92 \mathrm{~g} / \mathrm{h}$,), summer (CPUE; $0.89 \mathrm{n} / \mathrm{h}$, YPUE: $71.77 \mathrm{~g} / \mathrm{h}$ ) and spring (CPUE; $0.28 n / h$, YPUE: $24.63 \mathrm{~g} / \mathrm{h}$ ). The main reason for the given situation was the subnormal temperatures recorded in the winter season of the year 2017. These seasonal differences might stem from temperatures, abiotic factors such as flows, local migration, and biological factors such as abundant nutrition and bait selection. In addition, given factors, the condition of the fish, which may seasonally change, might be cause this differences.

In the context of handline fishing, fish behaviour set is classified within 4 phases as; the presence of bait, searching and findings of the bait, seizing and swallowing the bait (Fernö \& Huso 1983; Özdemir \& Erdem 2006). The main reason of selecting silicon mud shrimp for testing is to increase mud shrimps' duration of stay on the hook and increase their catching efficiency and catching performance. The most important senses for fishes to detect the bait are smell and visibility. Artificial mud shrimp (dummy mud shrimp) can be found in the market. This type of bait attracts a fish yet the lack of smell decreases its catching performance. Despite the fact that live mud shrimp catch higher number of individuals in handline fishing (13\%) in a unit of time $(1.57 \mathrm{n} / \mathrm{h})$ in comparison to silicone mud shrimp $(1.38 \mathrm{n} / \mathrm{h})$; in terms of weight of the product in a unit of time, silicone mud shrimp $(137.73 \mathrm{~g} / \mathrm{h})$ showed higher rates $(13 \%)$ in comparison to live mud shrimp bait $(121.84 \mathrm{~g} / \mathrm{h})$. Given the fact that the weight parameter identifies the price and level of economic revenue of the yield, it can be argued that the use of silicone mud shrimp bait is more profitable in handline fishing.

The most important benefit of the silicone mud shrimp in handline fishing was the decrease in the cost by allowing re-use of the bait (more than 5 times re-use than live mud shrimp). Another important contribution of this advantage was enabling to need lower amount of mud shrimps, particularly in the handline fishery. The given situation will offer a more environmentalist approach for catching, as fewer mud shrimps will be caught. In this study, mud shrimp's duration of staying on the hook was not calculated. On the other hand, it was observed that fishes hit the silicone mud shrimp bait to the point where there is only silicone left. The given situation allows the fishing line to stay longer in the water.

One of the most important disadvantages in experiments with live mud shrimp bait is providing of live mud shrimp particularly in the winter season. Mud shrimps bury in the mud in the cold-weather periods (winter) and continue their lives in deeper parts. This situation causes more difficult supplying live mud shrimp and its price increase 3 or 4 times than the normal season. Particularly in these periods, the use of silicone mud shrimp in handline fishing will help to decrease the cost of baits by preventing loses from the catches.

The captured of 590 individuals belonging to 30 species shows that the richness of the study area and the attractiveness of the selected baits. The maximum number of species in the Aegean Sea, in line fishing (including long line), was reached in this study (Kaykaç et al. 2003; Aydın 2011; Soykan \& Kınacıgil 2013). On the other hand, the results indicate that more than 70\% of the obtained individuals had economic significance and the seabream constituted $20 \%$ of the total catched products prove the importance of handline fishing in the region. The cephalopods had a low rate within the catch composition as $6.6 \%$. The main reason for the given situation is the methods that are used only for fish; and different handline and equipment are used for cephalopods (Beğburs et al. 2004; Kaykaç et al. 2012).

In terms of 3 baits of total catches, 9 species have sufficient data set for statistical tests. It was found that there is no significant difference between the lengths groups except two banded bream. Differences were found for two banned breams live mud shrimp-silicon pellet and silicon mud shrimp and silicone pellet. No difference was found for 15 species lengths groups between caught live and siliconized mud shrimp. The communication of the Republic of Turkey Ministry of Agriculture and Forestry No: 4/1, which regulated the commercial catch, imposed weight and lengths restriction species (RTMAF 2016). Among these, the minimum length sized for the species -which are also included in this study- have been announced as $20 \mathrm{~cm}$ for seabream, 18 $\mathrm{cm}$ for two banded bream and mackerel, $15 \mathrm{~cm}$ for common pandora and $21 \mathrm{~cm}$ for white seabream. The average total length of the seabreams obtained in experiments found as $17.62 \mathrm{~cm}$ (live mud shrimp bait: 17.17 , silicon mud shrimp bait: $18.07 \mathrm{~cm}$, which was below the legal length). While both common pandora and mackerel mean lengths were found above the minimum 
landing size. Differences were found for two banned breams, which were found different in terms of length groups of live mud shrimp-silicon pellet and silicon mud shrimp and silicone pellet.

There are no studies conducted on this type of bait which was used in the tests both in the Turkish and international literature, therefore direct comparisons could not be performed. There is only one study on the catching performance and catching efficiency of the mud shrimp (Erzini et al. 1998). The catching performance of razor shell (Ensis siliqua) and mud shrimp (Upogebia pusilla) were tested with round bend, flatted and spade end of 2316 DT hooks (numbers 11.13 and 15). It was found that the bait type did not significantly affect the catch size distribution. Although more fish were caught with the razor shell bait, higher catch rates red sea breams obtained with mud shrimp.

\section{Conclusions}

Article 32(b) of the communication (communication no: 2016/35) regarding the regulation of commercial fishery, provincial directorates have authorized to regulate catching method, time restriction, annual yield etc. for products to be used as bait in fisheries. According to this regulation, fishing, supplying and commercial sale of mud shrimp (including European razor clam, dye-murex and solitary tube worm) are forbidden within the borders of İzmir province between the dates of $1^{\text {st }}$ February and $30^{\text {th }}$ June. On the other hand, there are no records of statistics kept for this species, and of others, which are used as fish baits (European razor clam, solitary tubeworm etc.). It is stated that the catching pressure has been gradually increasing on this species, and the catching rates have been gradually decreasing. Therefore, population dynamics studies should be investigated.

A transparent silicone was used in the study. An important question is whether the silicon pellet which is commonly used in handline and long line fishing is digested by fish or not. There is no information on the individuals, which swallowed the silicone pellet. An urgent need to carry out studies on the questions of whether silicone pellet is digested (or defecated) or leads to death. In the case that any adverse impacts of silicon pellet will be detected, its commercial use should be forbidden.

The present study includes innovative ideas and methodologies to be used in handline fishing. Despite the fact that the number of individuals catches by mud shrimp in a unit of time in handline fishing is high, it was identified that the weight catches in a unit of time is more with a silicone mud shrimp. Given that the weight parameter is decisive in determining the price of a product and the level of economic input; it can be argued that silicone mud shrimp bait catching in handline fishing is more profitable. The study results lead to the conclusion that the use of silicone mud shrimp bait is useful particularly in the periods that a live mud shrimp cannot be used or provided.

\section{Acknowledgements}

The present study was sponsored by the Ege University Scientific Research Projects Fund (BAP Project No: 2015/SUF/025) and the Scientific and Technological Research Council of Turkey (Project No: TOVAG 215O241). We would like to thank both institutions for their support.

\section{References}

Akamca E \& Kiyağa V B (2009). Effects of live bait size on European seabass (Dicentrarchus labrax) angling. XV. National Fisheries Syposium, 01-04 July, Rize

Ateşşahin T, Duman E \& Cilbiz M (2015). Selectivity and catch efficiency of three spinner hook sizes in angling for rainbow trout (Oncorhynchus mykiss, Walbaum, 1792) in Karakaya Dam Lake (Eastern Turkey). Turkish Journal of Fisheries and Aquatic Sciences 15(4): 851-859 http://dx.doi.org/10.4194/1303-2712-v15_4_08

Aydın İ (2011). Is natural bait-type a stochastic process for size and condition of fishes in the recreational fishery from the İzmir Bay? Mediterranean Marine Science 12(2): 390-400 http://dx.doi.org/10.12681/mms.39

Beğburs C R, Altınağaç U \& Hoşsucu H (2004). A study on octopus handlines made from different materials (In Turkish). Ege Journal of Fisheries and Aquatic Sciences 21(1-2): 5-8

Erzini K, Gonçalves J M S, Bentes L, Lino G B \& Ribeiro J (1998). Species and size selectivity in a 'red' sea bream long line 'métier' in the Algarve (southern Portugal). Aquatic Living Resources 11(1): 1-11 http://dx.doi.org/10.1016/S0990-7440(99)80025-4

Fernö A \& Huse I (1983). The effect of experience on the behaviour of cod (Gadus morhua L.) towards a baited hook. Fisheries Research 2(1): 19-28 http://dx.doi.org/10.1016/0165-7836(83)90100-5

Froese R \& Pauly D (2019). FishBase. Retrieved in February, 12, 2019 from http://www.fishbase.org

Huse I \& Fernö A (1990). Fish behaviour studies as an aid to improved long line hook design. Fisheries Research 9(4): 287-279

Kaykaç M H, Arslan E, Göncüoğlu H, Maktay C B, Aydın C, Aydın İ, Bayramiç İ, Özdemir Y, Ünal V \& Tokaç A (2012). The Importance of the Color Factor and Squid (Loligo Vulgaris Lamarck, 1798) Fishing Methods in Izmir Bay (In Turkish). Ege University Scientific Research Projects Coordination, 2009/SÜF/023, İzmir

Kaykaç M H, Ulaş A, Metin C \& Tosunoğlu Z (2003). A study on catch efficiency of straight and kirbed hooks at handline fishing (In Turkish). Ege Journal of Fisheries and Aquatic Sciences 20(1-2): 227-231

Løkkeborg S (1991). Fishing experiments with an alternative long line bait using surplus fish products. Fisheries Research 12(1): $43-56$

Özdemir S \& Erdem Y (2006). Fish behaviour at fisheries by passive fishing gears (In Turkish). Ege Journal of Fisheries and Aquatic Sciences 23(1/3): 467-471

Palomares M L D \& Pauly D (2019). Sea Life Base. Retrieved in April, 23, 2019 from http://www.sealifebase.org 
RTMAF (2016). Notification 4/1 Regulating Commercial Fishing. Republic of Turkey, Minister of Agriculture and Rural Affair, General Directorate of Conservation and Inspection, Ankara

Soykan O \& Kinacigil H T (2013). Setlining for European Seabass (Dicentrarchus labrax, Linnaeus, 1758); technical features and bait preference. Ege Journal of Fisheries and Aquatic Sciences 30(2): 45-49 http://dx.doi.org/10.12714/egejfas.2013.30.2.01

TSMS (2017). Meteorological Dictionary-Turkish State Meteorological Service. Retrieved in August, 1 , 2019 from https://www.mgm.gov.tr/genel/meteorolojisozlugu.aspx

Zar J H (1974). Biostatistical Analysis. Englewood Cliffs, Prentice-Hall, New Jersey

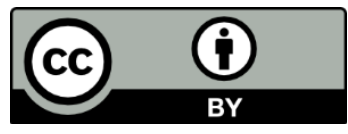

(C) 2021 by the authors. Licensee Ankara University, Faculty of Agriculture, Ankara, Turkey. This article is an open access article distributed under the terms and conditions of the Creative Commons Attribution (CC BY) license (http://creativecommons.org/licenses/by/4.0/). 